\title{
Emotional Responses And Perceived Stressors Of Frontline Medical Staffs In Case Of Addis Ababa Covid-19 Treatment Centers And Obstetrics Emergency And Abortion Care Clinic, Ethiopia; Analytical Cross Sectional Study
}

Mebratu Kebede ( $\square$ mebratuabraha21@gmail.com )

St. Paul's Hospital Millennium Medical College

Dereje Demissie

St. Paul's Hospital Millennium Medical College

Dessalegn Guddu

St. Paul's Hospital Millennium Medical College

Michael Haile

St. Paul's Hospital Millennium Medical College

\section{Zebenay Bitew}

St. Paul's Hospital Millennium Medical College

Mahteme Muleta

St. Paul's Hospital Millennium Medical College

\section{Research Article}

Keywords: Emotional responses, perceived stressors, front line medical staffs, Addis Ababa COVID-19 Treatment Centers

Posted Date: December 2nd, 2020

DOl: https://doi.org/10.21203/rs.3.rs-107059/v1

License: (c) (i) This work is licensed under a Creative Commons Attribution 4.0 International License. Read Full License 


\section{Abstract}

Background: the rapid spread of COVID-19, its lethality in severe cases and the absence of specific medicine poses a huge threat to human life and health, as well as huge impact on the mental health. Facing this critical situation, health care workers on the front line who are directly involved in the diagnosis, treatment, and care of patients with COVID-19 are at risk of developing psychological distress and other mental health symptoms including emotional disturbance.

Objective: the aim of this study will be to assess the current state of emotional responses and perceived stressors of frontline medical staffs in case of Addis Ababa COVID-19 Treatment Centers and obstetrics emergency and abortion care, Ethiopia 2020

Methods: Hospital based comparative cross-section study design was conducted by using selfadministered questionnaire survey from June $1^{\text {st }}$ to $30^{\text {th }}$ of 2020 among 133 and 266 frontline medical staffs from obstetric emergency and abortion care clinic and COVID-19 treatment centers respectively. The data were collected after getting written consent from each participant and it entered into the computer using Epi-data version 7, then exported to SPSS version 20 for further analysis. Descriptive analysis was done using frequencies \& percent. All independent determinants with P-value $<0.05$ were used to identify important predictors of emotional responses and perceived stressors.

Result; A total of 399 frontline medical staffs were included in the study. The mean age of the respondents of those who were working in obstetrics emergency and abortion care clinic was 27.47 $(S D=3.46)$ years and it was $28.12(S D=4.09)$ years for the other groups. This study revealed that, $72.9 \%$ and $5.6 \%$ of the study participant from obstetrics emergency and abortion clinic and COVID-19 treatment centers had a positive emotional response, respectively. Factors such as having a low level of motivational factors (AOR 2.78, 95\% Cl $(1.13,6.84)$ ), being a nurse (AOR 10.53, 95\% $\mathrm{Cl}(1.31,85.26)$ ) and working at triage (AOR $8.61,95 \% \mathrm{Cl}(1.15,64.81))$ ) had statistically significant association with negative emotional response.

Conclusion; The current study revealed that a high proportion of front line a negative emotional responses had negative emotional response. Further, almost all of the medical staffs working in COVID19 treatment centers and at obstetrics emergency and abortion care unit had perceived the outbreak related stressors. So, providing comprehensive psychological support is warranted for health care providers working in such kinds of department or units.

\section{Introduction}

Corona Virus Disease 2019 (COVID-19) is a disease caused by a novel corona virus (2019-nCoV) which was primarily identified in Wuhan, China on December, 2019 (1). It is highly infectious during the incubation period and can be transmitted from person to person through respiratory droplets, contact and aerosols (2). To date (October $16^{\text {th }}$ of 2020), more than 39,596,856.00 cases of COVID-19 are found globally, causing more than 1,107, 374.00 confirmed deaths around the globe (3). Due to the rapid spread 
of COVID-19, strong contagion, lethality in severe cases, and no specific medicine, it poses a huge threat to human life and health, as well as huge impact on the mental health of the general public like emotional disturbance (4).

Although infectious diseases elicit a wide range of emotional responses, not everyone experiences the same degree of emotional impact (5). However, health care providers who are directly involved in the diagnosis and treatment as well as care of patients with COVID-19 are at risk of developing psychological distress and other mental health symptoms (2). The ever-increasing number of suspected and confirmed cases, overwhelming workload as well as feelings of being inadequately supported, depletion of personal protection equipment and lack of specific drugs may all contribute to the mental burden like emotional disturbance of these health care workers. Previous studies have reported adverse psychological reactions to the 2003 SARS outbreak among health care workers (5-7).

Those health care workers feared contagion and infection of their close family experiencing high levels psychological distresses which could have long-term psychological implications (8). Unrecognized and asymptomatic patients transferred to their family or close contacts can be alarming and causing potentially distressing and emotional turmoil for front line health providers $(6,8)$. In general mental health problems are inevitable among front line health care providers(9-13). So, providing psychosocial support, timely psychological assistance and training in coping strategies to those frontline HCWs has its own contribution in regulating emotional response and perceived stressors $(14,15)$.

However, there are no published data that shows the emotions and perceived stressors of front line medical staffs in Ethiopia. To address this gap, the current study was aimed to assess the level of emotional response and perceived stressor among frontline Medical Staffs' from COVID-19 treatment centers and obstetrics emergency/abortion acre units in Addis Ababa city, Ethiopia.

\section{Methods}

\section{Study Area and period}

The study was conducted from June $1^{\text {st }}$ to $30^{\text {th }}$ of 2020 among 133 and 266 frontline medical staffs from obstetric emergency and abortion care clinic and COVID-19 treatment centers of Eka Kotebe General Hospital, St. Paul's Hospital Millennium Medical College (SPHMMC) and St. Peter specialized Hospital, Addis Ababa, Ethiopia. SPHMMC, a teaching Hospital located in the capital city, Addis Ababa. The current St. Peter 's hospital was established in 1963G.C as a TB treatment center in the nation. At the time of Haile Selassie, I, with the charity and goodwill of Knojit Anbenet, wife of Ras Abebe, the residence of the War Minister was given to Ministry of Health to serve as TB sanatorium. Eka Kotebe General Hospital in Addis Ababa has five-floor building that provides services of mental health with 150 beds and general medical services with 200 beds has been built with a total cost of 160 million Birr. A nine-floor building for administrative work and Mental Health Institute has also been inaugurated on the same day. Eka Kotebe General Hospital is under the auspices of the St Amanuel Mental Specialized Hospital. 


\section{Study Design, study population and inclusion criteria}

Hospital based comparative cross-sectional study was conducted among all frontline Medical Staffs who were working in Addis Ababa COVID-19 treatment Centers and obstetrics emergency and abortion care departments at least 2 weeks during the study period at Eka Kotebe General Hospital, St. Paul's and St. Peter Hospital in Ethiopia.

\section{Sample size determination, sampling technique, data collection and analysis}

Sample size was determined by using a single population formula considering the absence of previous data on most of the issues studied and to obtain the maximum sample size, this study assumed the proportion of stressors of frontline Medical Staffs to be $50 \%$ as p; and accordingly, the required sample size, $n$ at confidence interval of $95 \%$ with $5 \%$ degree of precision after adding a non-response rate of $10 \%$; the final sample size was 422 . The current research also allocates 1:2 ratio of front line medical staffs working at obstetrics emergency and COVID-19 treatment centers respectively. A simple random sampling technique was employed in order to select a representative sample of frontline Medical Staffs from each institution. Structured self-administered written questionnaires was administered to collect the data on the independent variables and outcome variables.

Emotional response was the extent that they experienced anxious, fear, sadness and anger in response to the outbreak of COVID-19 in in Addis Ababa COVID-19 treatment Center and obstetrics emergency and abortion care clinic on a 4-point scale, ranging from 0 (no such emotion) to 3 (the most intense feeling of the emotion). The questionnaire consisted of 15 questions that explored staff emotions during the COVID-19 outbreak. Finally, level of emotional responses was classified based on the participants score which $25 \%$ and above was considered as having a negative emotional response, whereas those who will score below $25 \%$ were considered as having a positive emotional response. With respect to perceived stressors, those who scored below $25 \%$ of the perceived stressor related question were considered as having no stressor and those who scored $25 \%$ and above were classified under having perceived stressor. The tool used to assess level of perceived stressor had also 4-point scale ( $0=$ very minimal; $1=$ slight; $2=$ moderate; $3=$ very much). Further social support status was measured based on Oslo 3 -item social support scale and those who scored greater than or equal to 9 (16).

Data was collected for one month, checked for completeness and consistency of responses manually. After cleaning it was entered, in to EPI-Data version 3.5.4 then exported to SPSS versions 25 for analysis. Bivariable and multivariable logistic analyses were performed to determine the presences of statistically significant associations between the independent variables and emotional responses and perceived stressor. All variables having a $p$-value $<0.25$ in the bivariable analysis were selected for the multivariable logistic regression to control for possible confounders. Those variables which showed significant association on bivariable analysis are adjusted to each other to identify independent determinants. The strength of the association was presented by odds ratio and $95 \%$ confidence interval. Variables with a pvalue of $<0.05$ on multivariable analysis were considered as statistically significant factors. 


\section{Ethical considerations}

Ethical approval and clearance was obtained from SPHMMC ethical review board and supportive letter was written to Eka General Hospital, SPHMMC, and St. Peter. In addition, consent of the respondents was obtained after giving information and thoroughly explaining the aim of the study to each respondent. The participants were told that participation is voluntary, could withdraw any time or refuse to answer to any question if they want to. The questionnaire was self-administrated questionnaire to maintain privacy. No information concerning the individual was passed to a third party.

\section{Results}

\section{Socio-demographic characteristics of the respondents}

A total of 133 and 266 frontline medical staffs from obstetrics emergency and abortion care clinic and COVID-19 treatment centers respectively were included in this study. The mean age of the respondents for those who were working in obstetrics emergency and abortion care clinic was $27.47(S D=3.46)$ years as well as it was $28.12(S D=4.09)$ years for the other groups. In the case of the respondents from obstetrics emergency and abortion care clinic, the majority 66 (49.6\%) were in age range of $26-30$ years, 94 (70.7\%) were male and 95 (71.4\%) were single. Further, 59 (44.4\%) were Resident/Intern in profession, 70 (52.6\%) \& 129 (97\%) were working at delivery unit and saint Paul hospital millennium medical college (SPHMMC) respectively. In addition, $112(84.2 \%)$ of them had no child, 88 (66.2\%) were not living with family at time of outbreak, $69(51.9 \%)$ had emergency obstetric/ abortion care treatment and prevention related training and $68(51.1 \%)$ had intermediate level of social support. (Table 1 )

Table1: - Socio demographic, professional and social support related description among frontline medical staffs in case of obstetrics emergency and abortion care clinic, Addis Ababa, Ethiopia, 2020. 


\section{Variables}

Frequency

Percent

(\%)

Age

25 years and

44

33.1

below

26-30 years

66

49.6

31 and above

23

17.3

years

Sex

Male

94

70.7

Female

39

29.3

Marital status

Married

38

28.6

Single

95

71.4

Place of work

Triage

6

4.5

Delivery unit

70

52.6

Abortion clinic

2

1.5

Obstetrics

operation room

5

3.8

Emergency

OBY/GNY

50

37.6

Profession

Nurse

9

6.8

Midwifery

42

31.6

Physician (GP)

6

4.5

Obstetric

Specialist

10

7.5

Gynecology

7

5.3

specialist

Resident/Inter

59

44.4

Clinical experience

2 years and below

80

60.2

3-4 year

25

18.8

5 year and above

28

21.1

Employer institution

YekaKotebe

2

1.5

Hospital

SPHMMC

129

97.0

St. peter Specialize

Hospital

2 


\begin{tabular}{|c|c|c|c|}
\hline \multirow[t]{2}{*}{ Have a child } & Yes & 21 & 15.8 \\
\hline & No & 112 & 84.2 \\
\hline \multirow[t]{2}{*}{ Living with family at time of outbreak } & Yes & 45 & 33.8 \\
\hline & No & 88 & 66.2 \\
\hline \multirow{2}{*}{$\begin{array}{l}\text { Have ever trained in emergency obstetric/ abortion } \\
\text { care treatment and prevention }\end{array}$} & Yes & 64 & 48.1 \\
\hline & No & 69 & 51.9 \\
\hline \multirow[t]{3}{*}{ Social support } & $\begin{array}{l}\text { Poor social } \\
\text { support }\end{array}$ & 53 & 39.8 \\
\hline & $\begin{array}{l}\text { Intermediate social } \\
\text { support }\end{array}$ & 68 & 51.1 \\
\hline & $\begin{array}{l}\text { Good social } \\
\text { support }\end{array}$ & 12 & 9.0 \\
\hline
\end{tabular}

With respect to the study participants in the case of COVID-19 treatment centers, the majority 137 (51.5\%) were in age range of $26-30$ years, 155 (58.3\%) were male and 177 (66.5\%) were single. Further, $219(82.3 \%)$ were Emergency medicine and Critical Care Specialist in profession, $143(53.8 \%)$ \& 170 $(63.9 \%)$ were working at clinical care unit and saint Paul hospital millennium medical college (SPHMMC) respectively. In addition, 195 (73.3\%) of them had no child, 148 (55.6\%) were not living with family at time of outbreak, 205 (77.1\%) had no COVID-19 treatment and prevention related training and 155 (58.3\%) had poor level of social support. (Table 2)

Table2: - Socio demographic, professional and social support related description among frontline medical staffs in case of COVID-19 treatment centers, Addis Ababa, Ethiopia, 2020. 


\section{Variables}

Age

31

Sex

Marital status

Place of work

Profession

Clinical experience

Employer institution

Have a child

Living with family at time of outbreak
Frequency

(\%)

31 years and above

Male

Female

Married

Single

Divorced/widowed

Critical Care Unit

Emergency Medicine

Outpatient Family Medicine

Ward

Triage

Emergency medicine and Critical

Care Specialist

Physician (GP)

Nurse

2 years and below

3-4 years

5 years and above

YekaKotebe Hospital

SPHMMC

St. peter Specialize Hospital

Volunteers

Yes

No

Yes

No
28.2

51.5

75

137

54

155

111

83

177

6

143

75

35

5

8

219

44

16.5

3

89

82

95

46

170

63.9

46

17.3

4

71

1.5

26.7

195

73.3

118

44.4

148

55.6 


\begin{tabular}{|llcc|}
\hline $\begin{array}{l}\text { Ever trained in COVID-19 treatment } \\
\text { and prevention }\end{array}$ & Yes & 205 & 77.1 \\
\hline Social support & No & 61 & 22.9 \\
\hline & Poor social support & 155 & 58.3 \\
\hline & Intermediate social support & 111 & 41.7 \\
\hline
\end{tabular}

\section{Level of emotional response among front line medical staffs}

This study revealed that, $72.9 \%(\mathrm{Cl} 66.2,80.5)$ and $5.6 \%(\mathrm{Cl} 3,8.3)$ of the study participant from obstetrics emergency and abortion clinic and COVID-19 treatment centers had positive emotional response respectively. (Fig.1) The Cronbach's alpha coefficient of emotional response categories of obstetrics emergency and abortion clinic and COVID-19 treatment centers are 0.704 and 0.743 respectively.

\section{Level of perceived stressor among the study participants}

This study revealed that the level of perceived stressor was $84.2 \%(\mathrm{Cl} 77.4,89.5)$ and $95.5 \%(\mathrm{Cl} 92.9$, 97.7) among frontline medical staffs working at obstetrics emergency and abortion care unit and at COVID-19 treatment centers respectively. The Cronbach's alpha result was 0.764 and 0.903 for perceived stressor of the study participants working at COVID-19 treatment centers and obstetrics emergency/abortion care units, respectively. (Fig.2)

\section{Factors associated with emotional response and perceived stressor among frontline medical staffs}

For each explanatory variable, bivariate analysis was done and factors such as place of working unit, profession and motivational status among frontline medical staffs working at obstetrics emergency and abortion care unit and factors such as social support and motivational factors among those who are working at COVID-19 treatment centers were variables fulfilled the minimum requirement at $p$-value $<0.05$ significance level for further multivariate logistic analysis of emotional response. Whereas emotional response was the only factor which fulfill the minimum requirement at $p$-value less than 0.05 during the bivariate analysis for further multivariate analysis with perceived stressor among health care providers working at obstetrics emergency and abortion care unit. But, there was no factor which had an association with perceived stressor during the bivariate analysis among those who are working at COVID19 treatment centers.

During the multivariate analysis for emotional response; factors motivational factors, profession and place of working unit had statistically significant associated with low level of emotional response among health care providers working at obstetrics emergency and abortion care unit; whereas only social support status had statistically significant association with emotional response among those frontline healthcare providers working at COVID-19 treatment centers. By using multivariate logistic regression, who had low level of motivational factors (AOR 2.78, 95\% $\mathrm{Cl}(1.13,6.84)$ ), nurses (AOR 10.53, 95\% $\mathrm{Cl}(1.31,85.26)$ ) and who were working at triage (AOR $8.61,95 \% \mathrm{Cl}(1.15,64.81))$ ) had statistically significant association with 
low level of emotional response among health care providers working at obstetrics emergency and abortion care units. Further, those who had low social support (AOR $8.02,95 \% \mathrm{Cl}(1.01,64.00)$ ) had statistically significant association with negative emotional response during the multivariate logistic regression among front line medical staffs working at COVID-19 treatment centers. (table 3)

Table 3; Factors associated with emotional response among frontline medical staffs working at obstetrics emergency/abortion care unit and COVID-19 treatment centers, Addis Ababa, Ethiopia, 2020 


\begin{tabular}{|c|c|c|c|c|c|}
\hline \multirow{2}{*}{$\begin{array}{l}\text { Explanatory } \\
\text { Variables }\end{array}$} & \multicolumn{2}{|c|}{ Emotional response } & \multirow[t]{2}{*}{ COR,95\%(Cl) } & \multirow[t]{2}{*}{ AOR,95\%(Cl) } & \multirow{2}{*}{$\begin{array}{l}\text { p- } \\
\text { value }\end{array}$} \\
\hline & $\begin{array}{l}\text { Positive } \\
\text { response }\end{array}$ & $\begin{array}{l}\text { Negative } \\
\text { response }\end{array}$ & & & \\
\hline \multicolumn{6}{|l|}{ Place of work $\left(a^{1}\right)$} \\
\hline Triage & 2 & 4 & $\begin{array}{l}7.09,(1.44 \\
43.96)^{*}\end{array}$ & $\begin{array}{l}8.61,(1.15 \\
64.81)^{\star *}\end{array}$ & 0.037 \\
\hline Delivery unit & 49 & 21 & $\begin{array}{l}1.52,(0.66 \\
3.53)\end{array}$ & $\begin{array}{l}1.50,(0.57 \\
3.95)\end{array}$ & 0.417 \\
\hline Abortion clinic & 2 & 0 & $\begin{array}{l}0.00,(0.00 \\
0.00)\end{array}$ & $0.00,(0.00,0.00)$ & 0.999 \\
\hline $\begin{array}{l}\text { Obstetrics operation } \\
\text { room }\end{array}$ & 5 & 0 & $\begin{array}{l}0.00,(0.00 \\
0.00)\end{array}$ & $\begin{array}{l}0.00,(0.00 \\
0.00)\end{array}$ & 0.999 \\
\hline Emergency OB/GYN & 39 & 11 & 1 & 1 & \\
\hline \multicolumn{6}{|l|}{ Profession( $\left.\mathrm{a}^{1}\right)$} \\
\hline OBY/GYN specialist & 15 & 2 & 1 & 1 & \\
\hline Nurse & 4 & 5 & $\begin{array}{l}9.38,(1.30 \\
67.65)^{*}\end{array}$ & $\begin{array}{l}\text { 10.53, }(1.31 \\
85.26)^{\star \star}\end{array}$ & 0.027 \\
\hline Midwifery & 26 & 16 & $\begin{array}{l}4.62,(0.93 \\
22.89)\end{array}$ & $\begin{array}{l}3.68,(0.68 \\
20.09)\end{array}$ & 0.132 \\
\hline Intern/GP/Resident & 52 & 13 & $\begin{array}{l}1.88,(0.38 \\
9.25)\end{array}$ & $\begin{array}{l}1.86,(0.35 \\
10.00)\end{array}$ & 0.470 \\
\hline \multicolumn{6}{|l|}{$\begin{array}{l}\text { Motivational level } \\
\left(a^{1}\right)\end{array}$} \\
\hline $\begin{array}{l}\text { low level of } \\
\text { motivation }\end{array}$ & 12 & 121 & $\begin{array}{l}2.13,(1.09 \\
4.69)^{\star}\end{array}$ & $\begin{array}{l}2.78,(1.13 \\
6.84)^{\star \star}\end{array}$ & 0.026 \\
\hline $\begin{array}{l}\text { high level of } \\
\text { motivation }\end{array}$ & 3 & 130 & 1 & 1 & \\
\hline \multicolumn{6}{|l|}{ Social support (b2) } \\
\hline $\begin{array}{l}\text { Poor/low social } \\
\text { support }\end{array}$ & 14 & 141 & $\begin{array}{l}\text { 10.92, } \\
(1.41,84.34)^{\star}\end{array}$ & $\begin{array}{l}8.02,(1.01 \\
64.00)^{\star \star}\end{array}$ & 0.049 \\
\hline $\begin{array}{l}\text { Intermediate social } \\
\text { support }\end{array}$ & 1 & 110 & 1 & 1 & \\
\hline \multicolumn{6}{|l|}{$\begin{array}{l}\text { Motivational status } \\
\left(\mathrm{b}^{2}\right)\end{array}$} \\
\hline $\begin{array}{l}\text { low level of } \\
\text { motivation }\end{array}$ & 12 & 121 & $\begin{array}{l}4.30,(1.18 \\
15.60)^{\star}\end{array}$ & $\begin{array}{l}0.36,(0.10 \\
1.36)\end{array}$ & 0.133 \\
\hline
\end{tabular}


* Significant association ( $p$-value $<0.05$ in bivariate) **-significant association ( $p$-value $<0.05$ in multivariate analysis);

$a^{1}$ - factors associated emotional response among HCP working at obstetrics emergency/abortion care unit

$\mathrm{b}^{2}$ - factors associated emotional response among front line medical staffs working at COVID-19 treatment centers

\section{Discussion}

Institution based comparative cross sectional study was conducted to assess the emotional responses, perceived stressors and coping strategies of frontline medical staffs in case of Addis Ababa COVID-19 treatment centers and obstetrics emergency and abortion care clinic, Ethiopia. The current study revealed that $72.9 \%$ of frontline medical staffs from obstetrics emergency and abortion clinic and $5.6 \%$ of those who are from Addis Ababa covid-19 treatment centers had positive emotional response. This implies health care workers working at COVID-19 treatment has more likely to have negative emotional response as compared to those who are working at obstetrics emergency and abortion clinic. That might be due to fear of acquiring the infection. Health professionals working at COVID-19 treatment center has high levels of burnout and psychological symptoms during the COVID-19 emergency which could have its own contribution to experience of negative emotion (17).

In other word $94.4 \%$ of the current study participants in the case of COVID-19 treatment centers had negative emotional response toward the outbreak which is higher than the previous study findings which was done in Italia indicated that $37 \%$ and $22.9 \%$ of the study participants had moderate and severe level of emotional exhaustion respectively (18). According to the other study which was conducted in the hospitals of the Istituto Auxologico Italiano, $35.7 \%$ and $31.9 \%$ had moderate and severe levels of emotional exhaustion that is markedly lower than the current finding(17).

High level of negative emotional response might have associated because of health professionals experiencing emotional contagion that perceive higher stress which over longing period can cause burnout. Emotional contagion and perceived stressor could increase the risk of burnout that could be expressed with negative emotion amongst health care providers (19).

In addition, the current study showed that $84.2 \%$ and $95.5 \%$ of frontline medical staffs working at obstetrics emergency and abortion care unit and at COVID-19 treatment centers respectively had perceived stressors. This indicated that, more or less, it was in line on health care providers working in both units. This is supported by the idea that globally, $90 \%$ of the HCP reported somewhat or substantially higher levels of stressors (20). However, the current study result was higher than the 
previous study finding reports such as in Wuhan more than half means $59.0 \%$ of the HCP had moderate to severe levels of perceived stress (21) and in India which 17.4\%, 78.9 and 3.7 (22), also 25.5\%, 22.9\% and $37 \%(18)$ had low, moderate and high level of perceived stressors respectively. Furthermore, it was also higher than the previous result that has been determined $54.5 \%$ of nurses and midwives have been making their lives worse since the outbreak started, $62.4 \%$ had difficulties in dealing with the uncertain situation in the outbreak (23). The variation might be due to the presence or absence of personal protective equipment's as well as other advanced medical infrastructures. The availability of strict infection control guidelines and specialized equipment provided psychological benefit especially in reduction stressors (24). Ongoing and old pressures from their personal life can affect the emotional expression of the HCP in their day to day job that their mood may change such as increased irritability and feel chronically exhausted that further persist as a stressor (25).

Regarding, the associated factors, the current study showed that those who were nurses were 10.53 times more likely to experience negative emotional response as compared to those OBY/GYN specialists. This is supported by the previous study findings which reported that being a nurse as predictor of emotional exhaustion $(17,24)$ and shows more affective symptoms $(18,26)$. So, timely counseling services and support systems should be given to those groups would help to mitigate the massive impact of the pandemic emergency on their actual and future emotional wellbeing (18).

With respect to place of working unit, those who were working at triage were 8.61 times more likely to have negative emotional response than those working at OBY/GYN emergency unit. This might be due to being as the first individual to be exposed with the patient and those who are working at the OBY/GYN emergency unit contact the patient after evaluated by the HCP working at triages. In addition, the current study identified that those who had low level of motivational factors were 2.78 times more likely to have negative emotional response as compared to those who had high level of motivational factors. This is augmented by the previous report that the presence of different motivating factors has a great contribution in reduction psychological impact like emotional disturbance of COVID-19 pandemic (24).

Among those front line health care providers working at COVID-19 treatment centers who had low social support were 8.02 times more likely to have negative emotional response than those who had good social support. This might be because of unavailability of proper support from their nearby individuals. The study which was done China mentioned that receiving negative feedback from families and friends contributes in the development of negative emotional response (27). Further, having better social support might serve as a buffer against the dysfunctional consequences of stress emanating from the workplace and established network of friends, family, superior, peers, and colleagues to seek emotional support when faced with job-related stress in the workplace (28) but it was in contrast with other finding that emotional response has no relation with presence or absence of family support (8).

\section{Conclusion}


The current study revealed that high proportion of front line medical staffs working at COVID-19 treatment centers $(94.4 \%)$ and at obstetrics emergency and abortion care unit $(27.1 \%)$ had negative emotional response. Further, almost all of the medical staffs working at COVID-19 treatment centers $(95.5 \%)$ and at obstetrics emergency and abortion care unit (84.2\%) had perceived the outbreak related stressors. Those health care providers who had low level of motivational factors, nurses, working at triage and had low social support were more experienced negative emotional response. So, providing comprehensive psychological support is warranted for those HCP working in such kinds of department or units.

\section{Declarations}

\section{Ethics approval and consent to participate}

The current manuscript had got ethical approval from Saint Paul's Hospital Millennium Medical College (SPHMMC) IRB as of the original research proposal which entitled "Investigate the current state of Emotional Responses, Perceived Stressors and Coping strategies of Frontline Medical Staffs in case of Addis Ababa COVID-19 Treatment Centers and obstetrics emergency and abortion care units, Addis Ababa, Ethiopia" and the principal investigator was Mr. Dereje Bayissa. So the current manuscript is reviewed and approved as being one of the specific objective which entitled "EMOTIONAL RESPONSES AND PERCEIVED STRESSORS OF FRONTLINE MEDICAL STAFFS IN CASE OF ADDIS ABABA COVID-19 TREATMENT CENTERS AND OBSTETRICS EMERGENCY AND ABORTION CARE CLINIC, ETHIOPIA; ANALYTICAL CROSS SECTIONAL STUDY" by Mr. Mebratu Abraha as corresponding author. In addition, informed consent was obtained from all study participants before they enrolled in to the study. They were told that their participation is voluntary, could withdraw any time or refuse to answer to any question if they want to. We were used self-administrated written questionnaire to maintain privacy. No information concerning the individual was passed to a third party. So, in general we carried out the current research by fulfilling all the requirements of the institutional (SPHMMC) IRB guidelines and regulations and also it fulfilled the Declaration of Helsinki as well as the relevant guidelines and regulations of BMC.

\section{Consent for publication}

Further, informed consent for publication was also obtained from each study participants under the consent form by mentioned for all of them that the data will be published in international journals. So, this is to confirm you that informed consent for publication was obtained from all the study participants. The collected data is kept confidentially under the primary investigator and co-investigators.

\section{Availability of data and materials}

Raw data were generated at Eka Kotebe General Hospital, SPHMMC and St.Petter Specialized Hospital. Derived data supporting the findings of this study are available from the corresponding author MA and co-author DB, ZW, DK, MT and MB on request. This is also to confirm you that there is hardcopy of ethical approval letter that we have got it from SPHMMC IRB committee after the research proposal had been 
reviewed and approved. The collected hardcopy questionnaires are available with the principal investigator DB, whereas the softcopy of SPSS data is currently available among some of the coinvestigators such as MA, ZW, MT, DK and MB by keeping it in confidential.

\section{Acknowledgements}

The authors acknowledge saint Paul Hospital Millennium Medical College IRB of the institution for their ethical evaluation and approval. The authors appreciate the respective study institution and the study participants for their cooperation in providing the necessary information.

\section{Authors' contributions}

MA was involved in developing the proposal, the study design, reviewed the article, analysis, report writing and drafted and write up the manuscript; DB was conceived the study and also involved in developing the proposal as a principal investigator, reviewing the manuscript; DK, MT, ZW \& MB were involved reviewing the manuscript.

\section{Funding}

There is no source of funding for the current manuscript, but the original research proposal which entitled "The current state of Emotional Responses, Perceived Stressors and Coping strategies of Frontline Medical Staffs in case of Addis Ababa COVID-19 Treatment Centers and obstetrics emergency and abortion care units, Addis Ababa, Ethiopia" which is done by Mr. Dereje Bayissa (currently mentioned as co-author) was sponsored by SPHMMC (Saint Paul's Hospital Millennium Medical College) academic and research vice provost office without including the allocated budget for publishing of the manuscript.

\section{Competing interest}

All authors read and approved the final manuscript. The authors declare that they have no competing interests.

\section{Authors' information}

Mr.Mebratu Abraha Kebede is an Assistant Professor of Integrated Clinical and Community Mental Health in his academic career, currently he is working at SPHMMC department of Nursing Education and Research Directorate office. He was a co-investigator (contributing on developing the original proposal, methods, final paper wring, data collection process and developing data collection instrument) during the original research work and he is the corresponding author of the current manuscript which submitted to your journal.

\section{References}

1. guidance WHOCmosariwNcniisi. Jan 11, 2020. 
2. Medicine SEGfCotEoNCPotCP. An update on the epidemiological characteristics of novel coronavirus pneumonia(COVID-19). Chinese Journal of Epidemiology. 2020;41(2):139-44.

3. WHO WC-OU. 2020.

4. Gao JL ZP, Jia YN, et al Mental Health Problems and Social Media Exposure during COVID-19 Outbreak. 2020.

5. Khalid I KT, Qabajah MR, et al Healthcare Workers Emotions, Perceived Stressors and Coping Strategies during a MERS-CoV Outbreak. Clinical Medicine \&Research. 2016;14(1):7-14.

6. Chiu HFK LL, Chiu E SARS andpsychogeriatric - perspective and lessons from Hong Kong. International Journal of Geriatric Psychiatry. 2003;18:871-3.

7. Leung PC OE. SARS War: Combating the Disease. New Jersey: World Scientific. 2003.

8. Lin CY PY, Wu YH, Chang J, Chan CH, Yang DY. The psychological effect of severe acute respiratory syndrome on emergency department staff. Emerg Med J. 2007 24:12-7.

9. Wong TW YJ, Chan CL, Kwong RS, Ho SM, Lau CC, et al. The psychological impact of severe acute respiratory syndrome outbreak on healthcare workers in emergency departments and how they cope. Eur J Emerg Med. 2005;12:13-8.

10. Folkman S LR, Pimley S, et al Agedifferencesin stress and coping processes. Psychology and Aging. 1987;2:171-84.

11. Folkman S LR. An analysis of coping inamiddle-aged community sample. Journal of Health and Social Behavior. 1980;21:219-39.

12. NH F. Emotions are functional, most of the time. In P. Ekman \& R. J. Davidson (Eds), The Nature of Emotion: Fundamental Questions New York: OxfordUniversity Press. 1994:112-22.

13. RW L. Human emotion: A functional view. In P. Ekman \& R. J. Davidson (Eds), The Nature of Emotion: Fundamental Questions. New York: Oxford University Press. 1994:123-6.

14. Long Huang FX, Hairong Liu. Emotional responses and coping strategies of nurses and nursing college students during COVID-19 outbreak 2020

15. D. H. Phua HKT, K. Y. Tham. Coping Responses of Emergency Physicians and Nurses to the 2003 Severe Acute Respiratory Syndrome Outbreak. ACAD EMERG MED. 2005;12(4).

16. Dalgard OS DC, Lehtinen V, Vazquez-Barquero JL, Casey P, Wilkinson G, Ayuso-Mateos JL, Page H, Dunn G; ODIN Group. Negative life event, social support and gender difference in depression. social psychiatry and psyciatric epidemiology. 2006;42(6):444-51.

17. Emanuele Maria Giusti EP, Guido E. D’Aniello, Chiara Stramba Badiale, Giada Pietrabissa, Chiara Manna, Marco Stramba Badiale, Giuseppe Riva, Gianluca Castelnuovo and Enrico Molinari The Psychological Impact of the COVID-19 Outbreak on Health Professionals: A Cross-Sectional Study. Front Psychol. 2020;11(1684).

18. Serena Barello LP, Guendalina Graffigna. Burnout and somatic symptoms among frontline healthcare professionals at the peak of the Italian COVID-19 pandemic. Psychiatry Research. 2020;290 (113129). 
19. Gunjan Joshi GS, , . Burnout: A risk factor amongst mental health professionals during COVID-19. Asian Journal of Psychiatry. 2020;54 (102300).

20. Aline Semaan CA, Elise Huysmans, Bosede Afolabi, Bouchra Assarag,Aduragbemi Banke-Thomas ,Hannah Blencowe, Séverine Caluwaerts, Oona Maeve Renee Campbell,Francesca L Cavallaro, Leonardo Chavane, et al. Voices from the frontline: findings from a thematic analysis of a rapid online global survey of maternal and newborn health professionals facing the COVID-19 pandemic. BMJ Global Health. 2020;5(002967).

21. Jiang Dua LD, Tao Wangc , Chenxin Yuana , Rao Fua , Lei Zhanga , Bo Liuc , Mingmin Zhangc, Yuanyuan Yinc, Jiawen Qinc, et al. Psychological symptoms among frontline healthcare workers during COVID-19 outbreak in Wuhan. General Hospital Psychiatry 2020.

22. William Wilson JPR, Seema Rao, Murtuza Ghiya, Nisanth Menon Nedungalaparambil, Harshit Mundra , Roshan Mathew, ... Prevalence and predictors of stress, anxiety, and depression among healthcare workers managing COVID-19 pandemic in India:A nationwide observational study. Indian J Psychol Med. 2020;42(4):353-8.

23. Yasemin Erkal Aksoya VK. Psychological effects of nurses and midwives due to COVID-19 outbreak: The case of Turkey,. Archives of Psychiatric Nursing. 2020:0883-9417.

24. Haozheng Cai BT, Jing Ma, Limin Chen, Lei Fu, Yongfang Jiang, Quan Zhuang. Psychological Impact and Coping Strategies of Frontline Medical Staff in Hunan Between January and March 2020 During the Outbreak of Coronavirus Disease 2019 (COVID 19) in Hubei, China. clinical research Med Sci Monit,. 2020;26( 924171 ):1643-3750.

25. Vijay Raghavan NJ, Akbar Ahmady Health Worker's Perception Survey on COVID 19 KNOWLEDGE, ATTITUDE AND PRACTICE ASSESSMENT IN EIGHT PROVINCES OF AFGHANSITAN,. The JOHANNIETR International Assistance. 2020.

26. Sofia Pappaa b, $\varangle, 1$, Vasiliki Ntellac, 1 , Timoleon Giannakasc, Vassilis G. Giannakoulisc, Eleni Papoutsic , Paraskevi Katsaounou, , . Prevalence of depression, anxiety, and insomnia among healthcare workers during the COVID-19 pandemic: A systematic review and meta-analysis. Brain, Behavior, and Immunity. 2020;88 901-7.

27. Jianyu Que LS, Jiahui Deng, Jiajia Liu, Li Zhang,Suying Wu, Yimiao Gong,Weizhen Huang, Kai Yuan,Wei Yan, Yankun Sun,Maosheng Ran, Yanping Bao, Lin Lu,. Psychological impact of the COVID-19 pandemic on healthcare workers: a cross-sectional study in China. General Psychiatry. 2020;33(100259).

28. Beh L-S. Job Stress and Coping Mechanisms among Nursing Staff in Public Health Services,. International Journal of Academic Research in Business and Social Sciences. 2012;2( 7 ):2222-6990

\section{Figures}




\section{Level of emotional response}

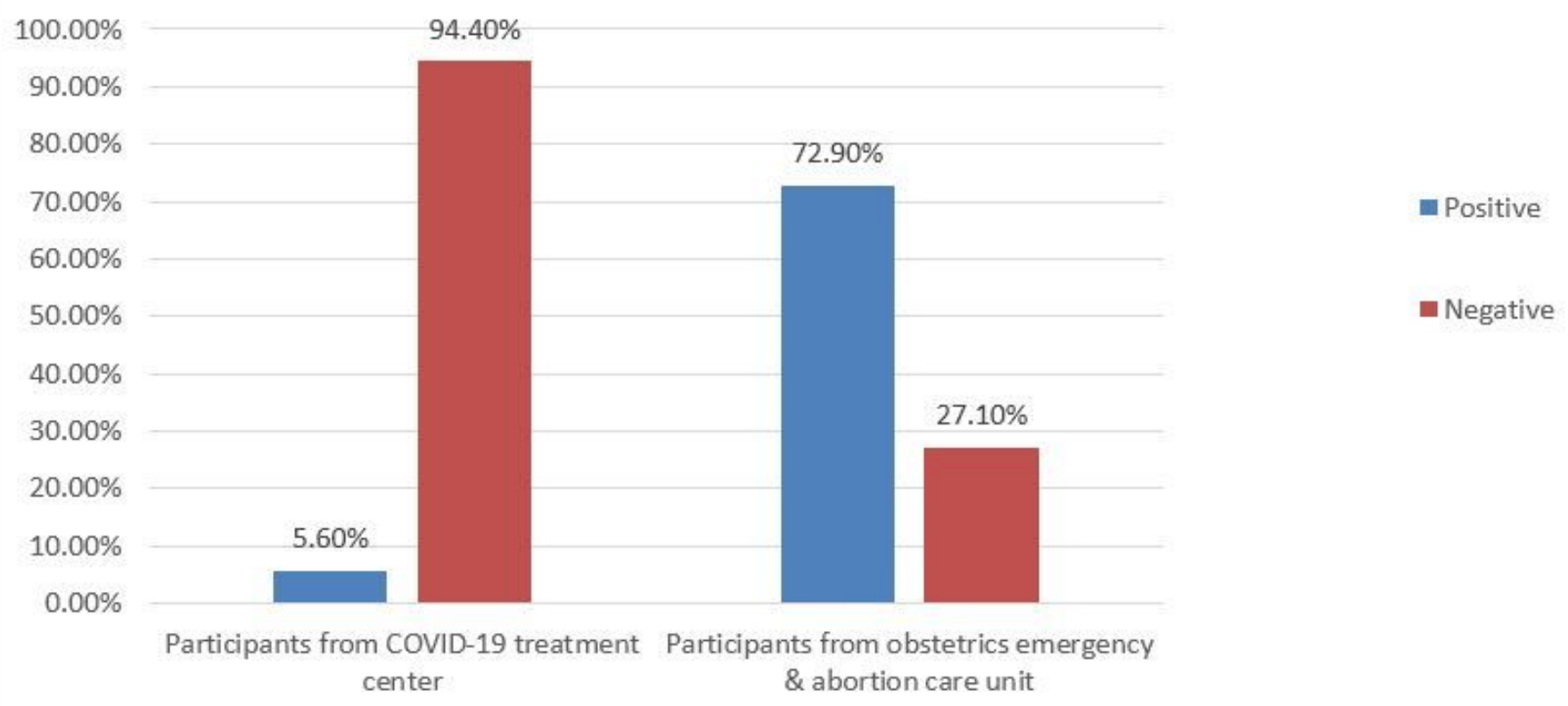

\section{Figure 1}

Level of emotional response among front line medical staffs working at obstetrics/abortion clinic and COVID-19 treatment centers, Addis Ababa, Ethiopia, 2020 


\section{Level of perceived stressor}

$120.00 \%$

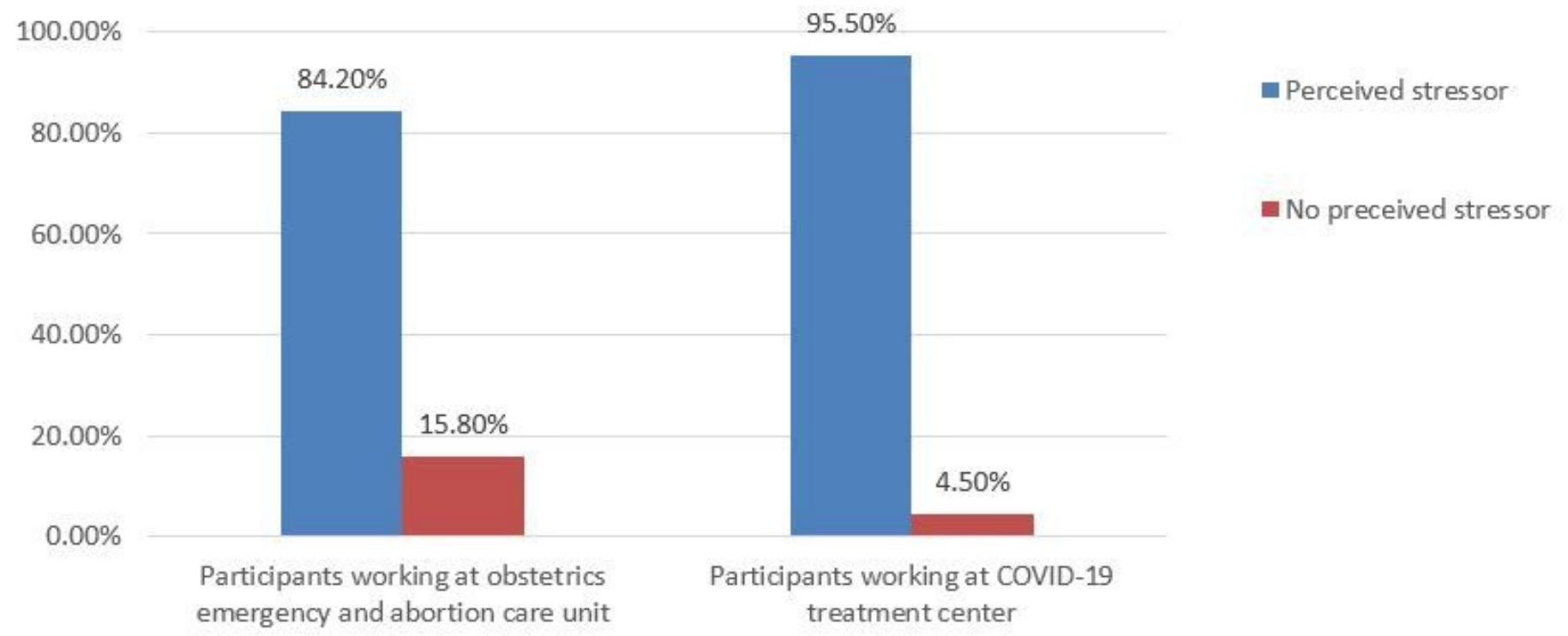

Figure 2

Level of perceived stressor status among front line medical staffs working at obstetrics/abortion clinic and COVID-19 treatment centers, Addis Ababa, Ethiopia, 2020

\section{Supplementary Files}

This is a list of supplementary files associated with this preprint. Click to download.

- Informnationsheetandconsentform.docx

- Informedconsentrevised11222020.docx 